\title{
Effect of Artificial Sweeteners on the Blood Glucose Concentration
}

\author{
${ }^{1}$ Bishamber D Toora, ${ }^{2}$ Seema S, ${ }^{3}$ Manju M, ${ }^{4}$ Sasmita Mishra
}

\begin{abstract}
Diabetes mellitus (DM) becoming pandemic and India is toward the capital of DM. Artificial sweeteners were extensively introduced into our diet with the intention of reducing caloric intake/ weight management. Several studies investigated its benefits as well as the health issues.
\end{abstract}

Aim: The present study was aimed to evaluate whether there is any change in blood sugar level after consuming artificial sweeteners. It also focused to determine artificial sweetener with less effect on blood sugar level.

Materials and methods: Healthy individuals $(n=30)$ of $18-25$ years with normal body mass index (BMI) $\left(19-25 \mathrm{~kg} / \mathrm{m}^{2}\right)$ and without any preexisting diseases were selected for the study. Fasting blood specimens and 1 hour after intake of glucose and artificial sweeteners, aspartame sucralose, stevia and saccharin (equivalent to $15 \mathrm{~g}$ of sugar with $200 \mathrm{~mL}$ of water) were collected. The significance of levels of glucose was studied with student's t-test and one-way analysis of variance (ANOVA).

Results: The mean fasting blood glucose concentration of all participants was in the range of $71.71 \pm 8.27$ to $80.94 \pm 7.30$ $\mathrm{mg} \%$. The mean glucose level after one $\mathrm{hr}$ of intake of glucose was $80.42 \pm 8.97 \mathrm{mg} \%$, and that of artificial sweeteners ranged from $74.42 \pm 8.34$ to $83.19 \pm 5.62$. A statistically significant decrease $(p<0.001)$ compared to the glucose intake has been observed in the difference in the glucose levels between the two samples. There was minimal increase in the blood glucose concentration after intake of the artificial sweeteners.

Conclusion: There was increase in the blood glucose level after the intake of artificial sweeteners and it was less compared to the glucose consumption. There was no difference in the values among the four artificial sweeteners, saccharine, aspartame, sucralose, and stevia. Stevia, being a natural product and having less side effect as compared to other artificial sweeteners, it can be suggested for incorporating as sugar substitute in dietary products.

Keywords: Artificial sweeteners, Aspartame, Saccharine, Sucralose, Stevia.

\footnotetext{
${ }^{1}$ Professor and Head, ${ }^{2}$ Tutor, ${ }^{3}$ Associate Professor, ${ }^{4}$ Professor

${ }^{1,2}$ Department of Biochemistry, Army College of Medical Sciences, New Delhi, India,

${ }^{3,4}$ Department of Biochemistry, Aarupadai Veedu Medical College, Puducherry, India
}

Corresponding Author: Seema S, Tutor, Department of Biochemistry, Army College of Medical Sciences, New Delhi, India, e-mail: seemasaraswathy09@gmail.com
How to cite this article: Toora BD, Seema S, Manju M, Mishra S. Effect of Artificial Sweeteners on the Blood Glucose Concentration. J Med Acad, 2018;1(2):81-85.

\section{Source of support: Nil}

Conflict of interest: None

\section{INTRODUCTION}

Recent past has observed an increased incidence of diabetes mellitus and metabolic syndrome. Obesity is suggested as one of the major causes of type II diabetes. The urbanization, changes in lifestyle and too much intake of fat may be the factors leading to obesity. ${ }^{1}$ The awareness of these data and consciousness of health and fitness have lead to the use of low-calorie sugar substitutes. Artificial sweeteners are alternatives to normally used sweeteners (sucrose) also known as non-nutritive sweeteners (NNS) with low calorie. ${ }^{2}$ They are commonly used in beverages and dietary products. The United States Food and Drug Administration authority has approved saccharine, aspartame, sucralose, neotame, acesulfame-K, and stevia for use in humans within the acceptable daily intake limit. ${ }^{1}$ In the present study, we have used saccharine, aspartame, sucralose, and stevia, which are commonly available in Indian market. The structure of these sweeteners are given in Figure 1.

Saccharin was the first artificial sweetener synthesized in 1879. It is a petroleum derivative and is about 200-700 times sweeter than sucrose. Saccharine is<smiles>C=C1CC23CCC4C(C)(C)CCCC4(C(=O)O)C2CCC1(O)CC3</smiles>

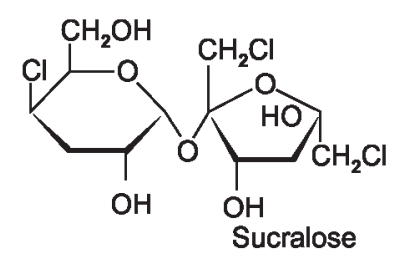<smiles>O=C1NS(O)(O)c2ccccc21</smiles><smiles>COC(=O)C(Cc1ccccc1)NC(CC(=O)O)CC(N)C(=O)O</smiles>

Saccharin

Fig. 1: Chemical structure of artificial sweeteners 
not being digested or metabolized. It is excreted via kidneys. ${ }^{3}$ Aspartame is a low-calorie sweetener and is 200 times sweeter than sucrose. ${ }^{4}$ It is aspartyl-phenylalanine-1-methyl ester (methyl ester of the dipeptide of the amino acids aspartic acid and phenylalanine). It is completely digested to its components, aspartic acid, phenylalanine, and ethanol..$^{5}$ The antipyretic, analgesic and anti-inflammatory action also have been shown by in-vitro studies. Sucralose is the non-caloric sweetener belongs to the group of organic chlorides. It is produced by chlorination of sucrose, by replacing three hydroxyl groups by chlorine. It is minimally absorbed. It is proved to be non toxic and fat-insoluble ${ }^{6}$ Stevia is extracted from leaves of South American plant, Stevia rebaudiana. Stevioside and rebaudioside are steviol glycosides which provide the sweetness. Stevioside has beta-D-glucose molecules replacing hydrogen atoms. ${ }^{7}$ Steviol glycosides are poorly absorbed in the body and pass through the upper gastrointestinal tract. On reaching the colon, gut bacteria hydrolyze steviol glycosides into steviol by removing glucose. Steviol is absorbed and metabolized in the liver forming steviol glucuronide and excreted in the urine. ${ }^{8}$ It is having no effect on blood pressure or blood glucose and it is proved to be non-allergic. Moreover, it satisfies the purity criteria established by The Joint FAO/WHO Expert Committee on Food Additives (JECFA, WHO). ${ }^{9}$

Notwithstanding the non-nutritive sweetness effect, studies were reported for the adverse health effects of artificial sweeteners. It is cited that continuous use of more than $1680 \mathrm{mg} /$ day leads to the risk of bladder cancer. Incidence in obesity had been seen in individuals with normal body mass index on the consumption of artificial sweeteners. In addition, an animal study showed the development of glucose intolerance by consuming saccharin. ${ }^{10}$ In another study, it is confirmed the allergic effect in sensitive individuals due to the intake of aspartame. It also has been suggested that it might be due to the diketopiperazine, a compound which forms when aspartame decomposes. Another significant finding was the formation of nitrosated compounds by combining the aspartame or its diketopiperazine breakdown product with nitrites in the diet. These compounds are identified to cause cancers. More so, the dose-dependent increase of lymphoma and leukemia in rats was demonstrated with aspartame. However, many other studies could not find any carcinogenic effect of aspartame. ${ }^{9}$

Medical complications like diabetes, cardiovascular disease, and cancer were documented in those consuming sucrose-sweetened beverages. ${ }^{11}$ Intake of products containing saccharin caused increased body weight and obesity in animals by unbalancing the homeostatic mechanism. ${ }^{12}$
High doses of stevia had an adverse effect on the reproductive system in animals. Besides, laboratory experiments showed the conversion of stevia to a mutagenic compound. However, within the acceptable daily intake (ADI) of $4 \mathrm{mg}$ / $\mathrm{kg}$ body weight, stevia is less toxic. ${ }^{7}$

From the foregoing, it is understood that there is an increase in the use of artificial sweeteners as sugar substitutes in dietary products. Various experiments were performed for analyzing its safety, toxicity and health hazards. Many investigations analyzed the effects of these substitutes in obesity, weight gain and metabolic syndrome. The direct effect of the artificial sweeteners on food intake, absorption, blood glucose/insulin values have not been explored well. Hence the present study aims to find the blood glucose level after the intake of four kinds of artificial sweeteners, saccharine, aspartame, sucralose, and stevia.

\section{MATERIALS AND METHODS}

The study was performed at the Aarupadi Veedu Medical College and Hospital, Pondicherry. Healthy individuals $(\mathrm{n}=30)$ of the age group 18-25 years with normal BMI $\left(19-25 \mathrm{~kg} / \mathrm{m}^{2}\right)$ and without any preexisting diseases were selected for the study. Subjects with any history of allergy or malabsorption were excluded. In addition, participants who were taking any dietary supplements were also not included in the study.

All the participants were given the instructions for a normal carbohydrate diet for 3 days before the investigation. After getting the informed consent and approval by the institutional ethics committee, $2 \mathrm{~mL}$ blood specimen from all the participants after a fast of 8-10 hours was collected between 7 AM and 8 AM. The blood samples were collected in vacutainers with sodium fluoride and potassium oxalate (1:3). Then, $15 \mathrm{~g}$ of anhydrous glucose in $200 \mathrm{~mL}$ of water was given. Fifteen grams is given to find out the change in blood glucose within the physiological limits. After one hour, 2 $\mathrm{mL}$ of blood samples were collected. During the period of the experiment, the participants were advised to be on rest and were restricted from any kind of physical activity. Plasma was separated from blood specimens, and glucose concentrations were estimated.

After one week, for all participants, artificial sweetener, Aspartame (INTAS biopharmaceuticals, Gujrat India) equivalent to $15 \mathrm{~g}$ of glucose (as per the instructions of the manufacturer) with $200 \mathrm{~mL}$ of water was given after collecting overnight fast blood specimen. Then after one hour, $2 \mathrm{~mL}$ of blood was collected. The procedure repeated with three other artificial sweeteners: Sucralose (India foods, Maharashtra, India), Stevia (Kanha Biogenic laboratories, HP, India) and Saccharin 
(Abbott India Ltd, Maharashtra, India) in the subsequent weeks and glucose concentration in two samples in each case were determined.

The concentrations of glucose were estimated by standard glucose oxidase (GOD) peroxidase (POD) method. GOD oxidizes glucose to gluconic acid and the hydrogen peroxide liberated is acted on by peroxidase enzyme to liberate nascent oxygen $(\mathrm{O})$. Nascent oxygen then couples with 4-amino antipyrine and phenol to form a pink colored quinoneimine dye. The absorbance was measured at $520 \mathrm{~nm}$ and compared with a glucose standard (100 mg\%) treated similarly.

The mean values of the glucose concentrations were calculated. The significance of fasting and 'after intake' level of glucose was studied with student's t-test. The difference in the blood glucose level between the two samples: fasting and after intake of glucose/artificial sweetener were analyzed for their significance by one-way ANOVA.

\section{RESULTS}

The mean fasting blood glucose concentration of all participants were in the range of $71.71 \pm 8.27$ to $80.94 \pm 7.30$ $\mathrm{mg} \%$. The mean glucose level after one hr of intake of glucose was $80.42 \pm 8.97 \mathrm{mg} \%$, and that of artificial sweeteners ranged from $74.42 \pm 8.34$ to $83.19 \pm 5.62$. The mean values of blood glucose levels have been given in Table 1 . A statistically significant decrease $(p<0.001)$ compared

Table 1: Mean values of glucose concentrations (Fasting and one hr after intake of various sweeteners)

\begin{tabular}{llll}
\hline Parameters & Group & Mean & + SD \\
\hline A. Fasting & Glucose & 72.23 & 9.53 \\
blood glucose & Aspartame & 71.71 & 8.27 \\
concentration (mg\%) & Sucralose & 78.55 & 8.37 \\
& Stevia & 73.16 & 5.29 \\
& Saccharin & 80.94 & 7.30 \\
\hline B. Blood glucose & & & \\
concentration after & Glucose & 80.42 & 8.97 \\
one hr of intake of & Aspartame & 74.42 & 8.34 \\
glucose/artificial & Sucralose & 81.26 & 8.77 \\
sweetener (mg\%) & Stevia & 75.55 & 5.28 \\
& Saccharin & 83.19 & 5.62 \\
\hline
\end{tabular}

Table 3: Percentage difference in values of glucose concentrations: (A) Fasting; (B) After intake of glucose and various sweeteners

\begin{tabular}{llcc}
\hline Parameters & Group & Mean & + SD \\
\hline Percentage & Glucose & $12.14^{\mathrm{b}}$ & 10.80 \\
difference & Aspartame & $3.90^{\mathrm{a}}$ & 4.48 \\
(A-B) & Sucralose & $3.50^{\mathrm{a}}$ & 3.56 \\
& Stevia & $3.60^{\mathrm{a}}$ & 8.49 \\
& Saccharin & $3.11^{\mathrm{a}}$ & 5.52 \\
\hline
\end{tabular}

a,b: Means with same superscript do not differ each other with in each parameter (DMR test) to the glucose intake has been observed in the difference in the glucose level between the two samples: fasting and after intake of glucose/artificial sweetener (Table 2). There was no intergroup variation in values noticed as per the Duncan's multiple range test. The percentage difference is shown in Table 3. Though it is statistically significant, there is only a minimal increase in the after intake glucose concentrations for the artificial sweeteners (Table 4). Graph 1 shows the comparison of glucose concentrations of fasting specimen and after intake of various sugars. The mean difference between the fasting glucose concentrations and after intake of glucose and artificial sweeteners is illustrated in Graph 2.

\section{DISCUSSION}

Artificial sweeteners/sugar substitutes are food additives used instead of table sugar for reducing the calorie intake/ weight management, and it enhances taste also. Besides, it is therapeutically used in controlling the blood glucose, in preventing dental caries and in reactive hypoglycemia. Recently, the intake of these has been tremendously increased. In spite of these advantages, there are various health hazards also have been reported for artificial sweeteners. Different types of health issues like, cancers, metabolic syndromes, weight gain, etc. have been studied.

In the present study, the direct effect of artificial sweeteners, saccharine, aspartame, sucralose, and stevia on blood glucose levels have been analyzed. The concentrations of blood glucose after one hour of intake of these four sweeteners were detected. The values obtained were compared with that of glucose intake. As the healthy individuals were selected for the study, the

Table 2: Difference in mean values of glucose concentrations: (A) Fasting; (B) After intake of glucose and various sweeteners

\begin{tabular}{lllll}
\hline Parameters & Group & Mean & $+S D$ & F value \\
\hline & Glucose & $8.19^{\mathrm{b}}$ & 5.95 & \\
Difference & Aspartame & $2.71^{\mathrm{a}}$ & 3.10 & \\
$(\mathrm{~A}-\mathrm{B})$ & Sucralose & $2.71^{\mathrm{a}}$ & 2.76 & $8.796^{* *}$ \\
& Stevia & $2.39^{\mathrm{a}}$ & 6.44 & \\
& Saccharin & $2.26^{\mathrm{a}}$ & 4.49 & \\
\hline${ }^{* *} p<0.001 ;{ }^{\text {a,b: }}$ : Means with same superscript do not differ each other \\
with in each parameter (DMR Test)
\end{tabular}

Table 4: Comparison of the glucose concentrations of (A) Fasting; (B) after intake of glucose and various sweeteners by student's $\mathrm{t}$ test

\begin{tabular}{llll}
\hline Group & FBS (A) & After intake (B) & p value \\
\hline Glucose & $72.23^{\mathrm{a}}$ & $80.42^{\mathrm{b}}$ & $7.664^{* *}$ \\
Aspartame & $71.71^{\mathrm{a}}$ & $74.42^{\mathrm{a}}$ & $4.866^{* *}$ \\
Sucralose & $78.55^{\mathrm{b}}$ & $81.26^{\mathrm{b}}$ & $5.468^{* *}$ \\
Stevia & $73.16 \mathrm{a}$ & $75.55 \mathrm{a}$ & $2.064^{*}$ \\
Saccharin & $80.94^{\mathrm{b}}$ & $83.19^{\mathrm{b}}$ & $2.797^{* *}$ \\
\hline${ }^{* *} p<0.001 ;$;,b. Means with same superscript do not differ each other \\
with in each parameter (DMR test)
\end{tabular}




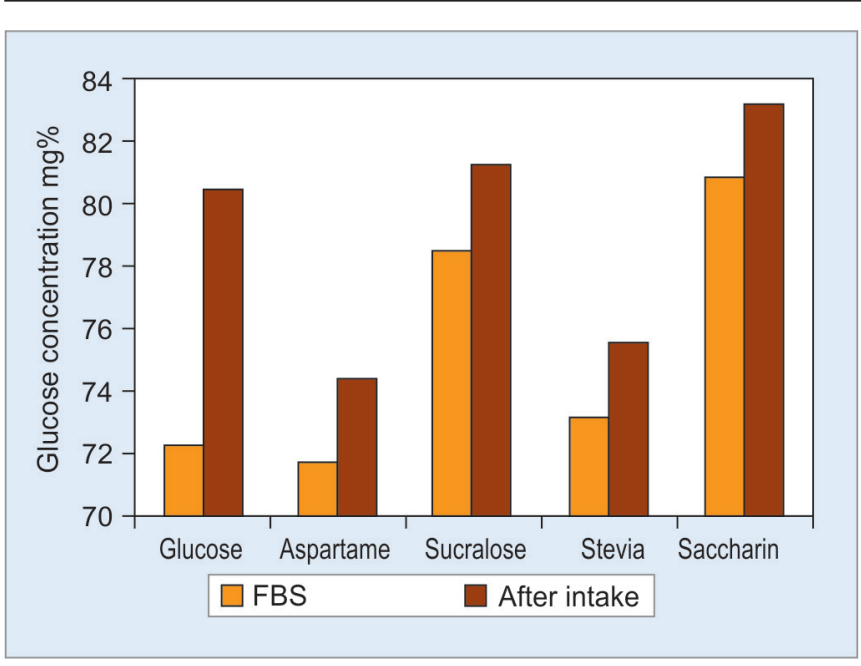

Graph 1: Comparison of FBS and after intake of artificial sweeteners (mg\%)

fasting values were within the normal range $71.71 \pm 8.27$ to $80.94 \pm 7.30 \mathrm{mg} \%$. The blood glucose values after the intake ranged from $74.42 \pm 8.34$ to $83.19 \pm 5.62$. The second samples, i.e., after the intake of artificial sweeteners showed a minimal increase, $2.26-2.71 \mathrm{mg} \%$. There was no difference detected between the different groups. Statistically significant difference in these values were noted when compared with glucose intake values $(p<0.001)$. Similar to this, lower postprandial values of aspartame and stevia were reported compared to sucrose intake by Anton SD et al. ${ }^{13}$ They suggest the effects may be due to the lower calorific and carbohydrate intake compared to sucrose. They also cite that some other factors may are also be involved in the decrease. In our study also, the similar decline in blood glucose values was shown by all four artificial sweeteners as compared with glucose value.

Though it is statistically significant, there is only a marginal increase in the blood glucose concentration after intake of saccharine, aspartame, sucralose, and stevia. There are different mechanisms by which artificial sweeteners are metabolized in the body. Some studies concluded that sucralose is not metabolized in the body whereas some reports that they are partially broken down in the gut. ${ }^{14,15}$ Aspartame is catabolized to their respective component amino acids. The increase of blood glucose by intake of aspartame needs further investigations. The findings of the low blood glucose level can be supported with the low insulin secretion followed by aspartame intake. ${ }^{16}$ For stevia also, the level of secretion of insulin after consumption was low compared to sucrose. Saccharine is not metabolized in the body. However, the insulin release after the intake was reported. ${ }^{16}$

The intake of artificial sweeteners reduced blood glucose and insulin levels. As far as health risks are concerned, experiments demonstrate risks for health, but the results are not conclusive. However, stevia being

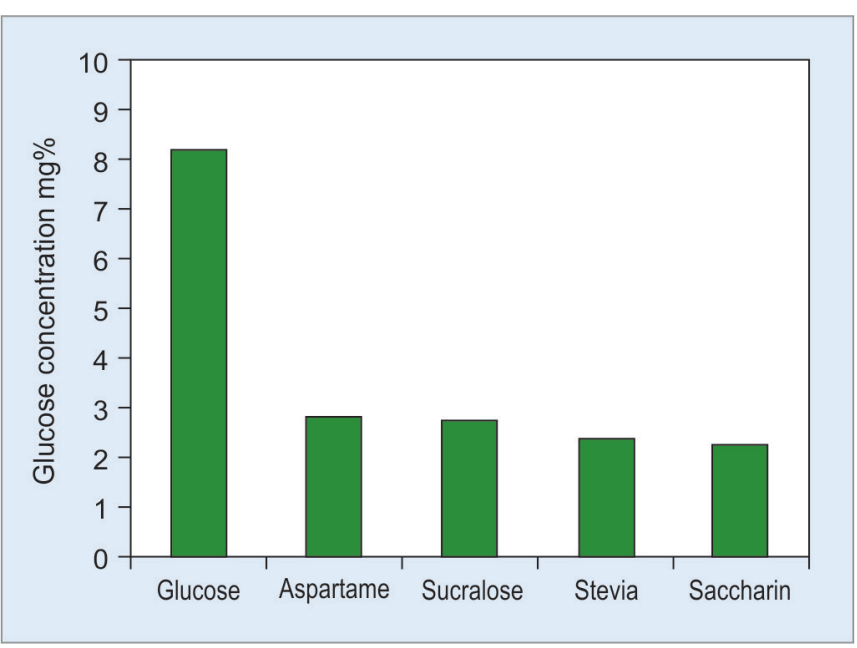

Graph 2: Mean difference between FBS and after intake of artificial sweeteners ( $\mathrm{mg} \%$ )

a natural product and with a minimal side effect, can be considered as a better sugar substitute.

\section{CONCLUSION}

The present study evaluated the effect of artificial sweeteners on blood glucose levels. The increase in blood glucose levels after the intake of artificial sweeteners was minimal compared to glucose consumption. There was no difference in the values among the four artificial sweeteners, saccharine, aspartame, sucralose, and stevia. Additional experiments are required to elucidate the exact mechanisms of absorption, and excretion of the artificial sweeteners.

\section{REFERENCES}

1. Sharma A, Amarnath S, Thulasimani M, Ramaswamy S. Artificial sweeteners as a sugar substitute: Are they really safe? Indian J Pharmacol. 2016;48(3):237-240.

2. Lange FT, Scheurer M, Brauch HJ. Artificial sweeteners-a recently recognized class of emerging environmental contaminants: a review. Analytical and Bioanalytical Chemistry. 2012;403(9):2503-2518..

3. Okoduwa SIR, Ebiloma GU, Baba J, Ajide S. The metabolism and toxicology of saccharin. Info health awareness article. 2013;1(1):14-19.

4. Kroger M, Meister K, Kava R. Low calorie sweetners and other sugar substitutes: A review of the safety issues. Compr Rev Food Sci Food Saf. 2006;5:35-47.

5. Lin SY, Cheng YD. Simultaneous formation and detection of the reaction product of solid-state aspartame sweetener by FT-IR/DSC microscopic system. Food Additives and Contaminants. 2000;17(10):821-827.

6. Sims J, Roberts A, Daniel JW, Renwick AG. The metabolic fate of sucralose in rats. Food and chemical Toxicology. 2000;38:115-121.

7. Geuns JM. Stevioside. Phytochemistry. 2007;64:913-921.

8. Prakash I, Chaturvedula VS. Steviol Glycosides: Natural Non-Caloric Sweeteners. Sweeteners: Pharmacology, Biotechnology, and Applications. 2017:1-28. 
9. Tandel KR. Sugar substitutes: Health controversy over perceived benefits. J Pharmacol Pharmacother. 2011; 2(4):236-243.

10. Renwick AG. The intake of intense sweeteners-An update review. Food Addit Contam. 2006;23:327-338.

11. Gardner C, Wylie-Rosett J, Gidding SS, Steffen LM, Johnson RK, Reader D, et al. Nonnutritive sweeteners: Current use and health perspectives: A scientific statement from the American Heart Association and the American Diabetes Association. Diabetes Care. 2012;35:1798-1808.

12. Hampton T. Sugar substitutes linked to weight gain. JAMA. 2008;299:2137-2138.

13. Anton SD, Martin CK, Han H, Coulon S, Cefalu WT, Geiselman $\mathrm{P}$, et al. Effects of stevia, aspartame, and sucrose on food intake, satiety, and postprandial glucose and insulin levels. Appetite. 2010;55(1):37-43.

14. Schiffman SS, Rother KI. Sucralose, a synthetic organochlorine sweetener: Overview of biological issues.

15. Schiffman SS, Abou-Donia MB. Sucralose revisited: Rebuttal of two papers about Splenda safety. Regulatory toxicology and pharmacology. 2012;63(3):505-508.

16. Malaisse WJ, Vanonderbergen A, Louchami K, Jijakli H, Malaisse-Lagae F. Effects of artificial sweeteners on insulin release and cationic fluxes in rat pancreatic islets. Cell Signal. 1998;10(10):727-733. 